RESEARCH ARTICLE

\title{
Lessons learned from the virus indexing of Musa germplasm: insights from a multiyear collaboration
}

\author{
C. De Clerck ${ }^{1, \dagger}$, K. Crew ${ }^{2, \dagger}$, I. Van den houwe ${ }^{3}$, L. McMichael ${ }^{2}$, C. Berhal ${ }^{1}$, L. Lassois ${ }^{1}$, \\ M. Haissam Jijakli ${ }^{1}$, N. Roux ${ }^{4}$, J. Thomas ${ }^{5}$ \& S. Massart ${ }^{1}$ \\ 1 Laboratory of Integrated and Urban Phytopathology, Gembloux Agro-Bio Tech, University of Liège, Gembloux, Belgium \\ 2 Queensland Department of Agriculture and Fisheries, Brisbane, Australia \\ 3 Bioversity Transit Centre, c/o Laboratory of Tropical Crop Improvement, Leuven, Belgium \\ 4 Bioversity International, Commodity Systems and Genetic Resources Programme, Montpellier Cedex, France \\ 5 The University of Queensland, Queensland Alliance for Agriculture and Food Innovation, Ecosciences Precinct, Brisbane, Australia
}

\section{Keywords}

Banana viruses; diagnostic test; virus indexing; phytosanitary certification; molecular diagnostic; electron microscopy; virus miniprep

\section{Correspondence}

S. Massart, Laboratory of Integrated and Urban Phytopathology, Gembloux Agro-Bio Tech, University of Liège, Passage des déportés, 2, 5030 Gembloux, Belgium. E-mail:

sebastien.massart@ulg.ac.be; and J. Thomas, The University of Queensland, Queensland Alliance for Agriculture and Food Innovation. Ecosciences Precinct, GPO Box 267, Brisbane, Queensland 4001, Australia.E-mail: j.thomas2@uq.edu.au

${ }^{\dagger}$ Equal first authors.

Received: 17 November 2016; revised version accepted: 10 January 2017.

doi:10.1111/aab.12353

\begin{abstract}
The Bioversity International Transit Center (ITC) for banana hosts more than 1500 accessions largely covering the genetic diversity of the genus Musa. Its objective is to conserve this genetic diversity and to supply plant materials to users worldwide. All the Musa accessions must be tested for virus presence and, if infected, virus elimination must be attempted, to enable the supply of virus-free plant material. An international collaborative effort launched under the auspices of Bioversity International (2007-2013) finally led to the implementation of a two-step process to test the accessions. The first step, called pre-indexing, involved only molecular tests and was designed as a pre-screen of new germplasm lines or existing accessions to reduce the need for post-entry virus therapy and repeated virus indexing. The second step, called full indexing, was performed on either older existing accessions or newer accessions which tested negative during pre-indexing, and involved molecular tests, transmission electron microscopy (TEM) and symptom observation. In total, 270 germplasm lines (434 samples) were pre-indexed; while full indexing was carried out on 243 accessions (68 of which had been pre-indexed). A significant proportion of the samples tested during pre-indexing was infected with at least one virus $(68 \%)$, showing the utility of this early pre-screening step. Banana streak $O L$ virus and Banana mild mosaic virus were the most commonly detected viruses during both pre- and full indexing. For 22 accessions, viral particles were observed by TEM in full indexing while the molecular tests were negative, underlining the importance of combining various detection techniques. After full indexing, viruses were not detected in 166 accessions, which were then released for international distribution from the ITC. This publication exemplifies how the practical application of diagnostic protocols can raise fundamental questions related to their appropriate use in routine practice and the need for their continuous monitoring and improvement after their first publication.
\end{abstract}

\section{Introduction}

Banana is a perennial herbaceous plant and is important for food security, feeding millions of small holders, and as a cash crop in many developing countries. Musa acuminata Colla subspecies (A genome) and M. balbisiana Colla (B genome) are the ancestors of the parthenocarpic Musa diploid and polyploid cultivars grown around the world. In 2012, they were grown on an area of 10.4 million ha worldwide, of which $48 \%$ was for dessert banana and $52 \%$ for cooking bananas, including plantain (FAO, 2014). They are among the 10 most important staple food crops worldwide with a global production of 139.5 million 
tonnes in 2012 (FAO, 2014). About 85\% of this global production comes mostly from small plots and kitchen or backyard gardens in the developing world, while the 15\% which is exported comprises mostly Cavendish subgroup dessert banana grown in large scale plantations (Ortiz \& Swennen, 2014).

As a response to the increasing threats to banana production in numerous countries, particularly through the spread of black leaf streak disease (caused by Mycosphaerella fijiensis) in the early 1980s, Bioversity International established a collection that has over the years become the most comprehensive repository of Musa genetic resources in the world. Managed by the ITC of Bioversity International, this Food and Agriculture Organization (FAO) 'in trust' collection plays a key role at a global level as a large proportion of the crop known genepool is safeguarded in perpetuity.

Presently, the collection comprises more than 1500 accessions, covering a broad range of cultivated forms belonging to 17 genome groups and 52 subgroups, representatives of 29 wild species and subspecies, and banana varieties improved through conventional breeding. Introduced from 57 sources in 37 countries, accessions from major field collections and Bioversity International collecting initiatives in the centre of origin (South East Asia) and secondary centres of diversity (West and East Africa) of the crop, and from major banana breeding programmes worldwide are safely duplicated in the ITC collection. The collection is also recognised as the most extensive source of healthy germplasm that is easily and freely accessible for use (Garming et al., 2010). Since 1985, more than 17000 samples of accessions were supplied to users' organisations in 103 countries, serving as resources for basic and applied research, breeding, conservation and a wide range of development activities.

Collection, conservation and utilisation of plant genetic resources and their global distribution are essential components of international crop improvement programmes. Guaranteeing the movement of pathogen-free germplasm is an important task to minimise the risk of pest introduction to new countries. In particular, pathogens that may sometimes be symptomless, such as viruses, pose a special risk to the movement of vegetative germplasm.

Some economically important viruses of banana have a limited international distribution, underlying the importance of distributing only virus-free material. For example, Banana bract mosaic virus (BBrMV) is currently only present in some countries in Asia and the Pacific (Fig. 1), and Banana bunchy top virus (BBTV) is present in some countries in Africa and Asia but absent in mainland America (Kumar et al, 2015; Thomas et al., 2015). The eradication of an introduced pathogen is indeed extremely difficult, or even impossible, and requires significant resources. A well-documented example is the BBTV eradication programme launched in Australia (Geering, 2009; Cook et al, 2012; Blomme et al., 2013). To minimise the risk of introduction, preventive measures and effective testing procedures are required to ensure that distributed material is free of pests of potential phytosanitary importance. A particularly problematic situation concerns the presence of infective endogenous banana streak viruses (eBSV) in most Musa cultivars containing the B genome (Duroy et al., 2016). The eBSVs can escape the host genome spontaneously (activation) following stress conditions such as use of in vitro culture or interspecific breeding processes, leading to vertical transmission of an episomal infection (Iskra-Caruana et al., 2014). Detection of these viruses thus requires assays which distinguish episomal from endogenous viral sequences.

Within the framework of the Bioversity International effort for safe germplasm distribution, an international collaboration was set up to complement the ongoing virus indexing of Musa germplasm with the eradication of detected viruses in the infected germplasm through sanitation processes (virus elimination or therapy). This publication summarises the output and conclusions of this collaboration over a 7-year period (2007-2013).

This effort finally led to the implementation of a two-step process, combining the targeted molecular detection of the main viruses of the genus Musa [BBTV, BBrMV, Banana mild mosaic virus (BanMMV), six Banana streak viruses (BSV) and Cucumber mosaic virus (CMV)] with symptom observation and TEM to detect the presence of any virus(es). Chemotherapy and thermotherapy of infected accessions were used to eliminate virus(es), details of which will be published separately.

Under the auspices of the FAO and the International Plant Genetic Resources Institute (IPGRI), technical guidelines have been published, describing procedures to minimise the risk of pest introductions due to the movement of germplasm for research, crop improvement, plant breeding, exploration or conservation. A first version of these guidelines was published in 1989 (Frison \& Putter, 1989) and was superseded by a second version in 1996 (Diekmann \& Putter, 1996). A new version of these guidelines (Thomas et al., 2015) has been produced by the Conservation Thematic Group of MusaNet, an international network for Musa genetic resources coordinated by Bioversity International. These guidelines make general and technical recommendations on safe procedures to move banana germplasm and focus on the most relevant aspects of virus biology and on their worldwide distribution as well as on the techniques available to detect them. 

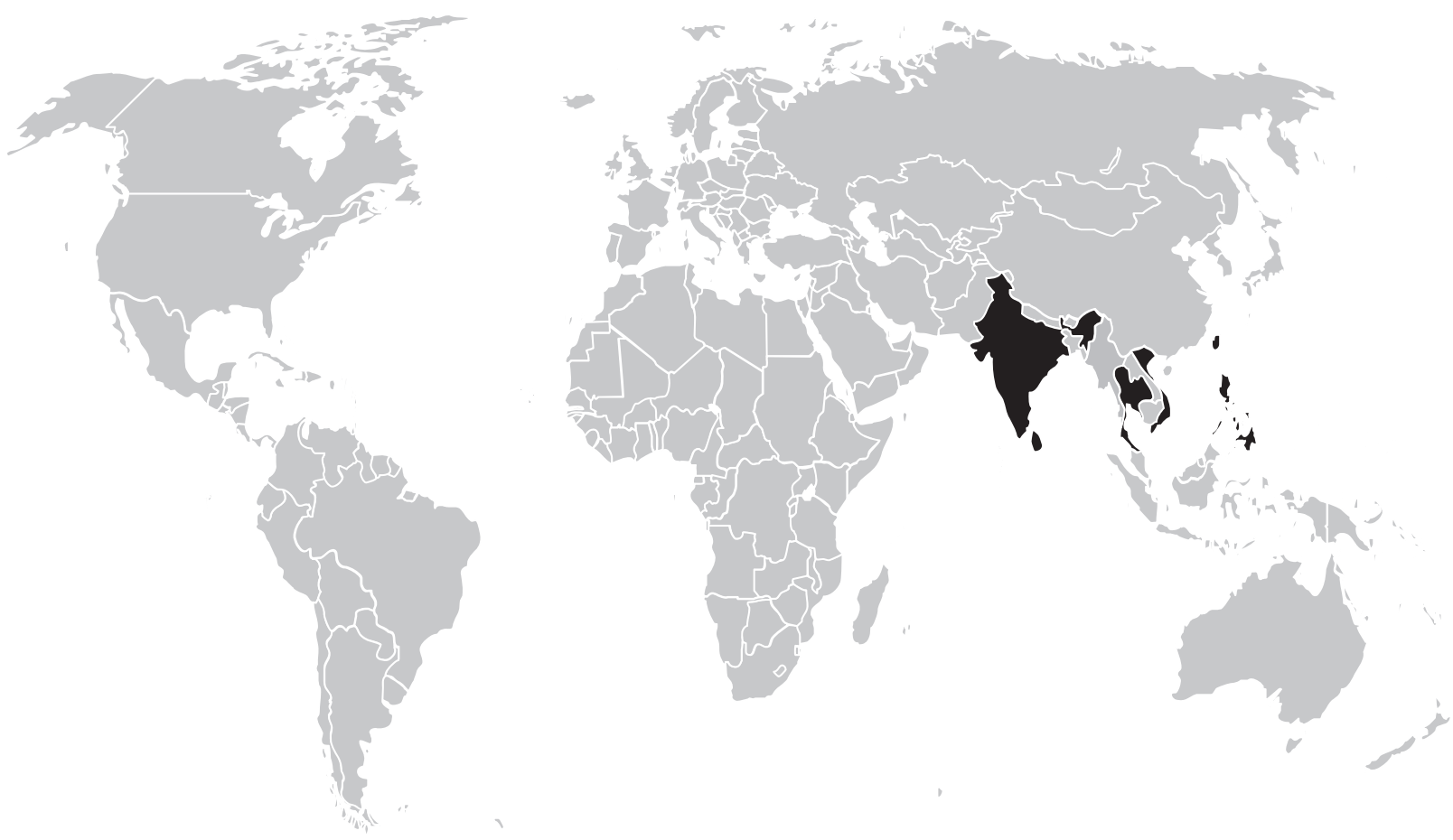

Figure 1 Worldwide distribution of Banana bract mosaic virus (BBrMV). Countries where the virus has been reported are in black.

These guidelines have been revised, based on the scientific knowledge accumulated and on the technological improvement in diagnostic protocols since 1996 but also in the learnings gained from the routine indexing of Musa germplasm collection managed by Bioversity International since the early 1990s. This article aims to give the scientific background and rationale which has led to some important recommendations in the technical guidelines. The vast majority of publications concerning diagnosis of plant pathogens are focused on the technical development of innovative protocols based on new technological advances. They show improvement of specificity, sensitivity, robustness or rapidity of the diagnostic assay or make it more user-friendly. These developments have a great potential for improving the diagnosis of plant pathogens but their performance in routine analyses is very rarely reported. After its technical development, a new diagnostic technique should be first validated through an inter-laboratory evaluation (Massart et al., 2008, 2009). Moreover, it must be adequately integrated into routine practice with an appropriate sampling protocol and its performance should be regularly monitored.

The results detailed in this paper show how the practical application of diagnostic protocols raises fundamental questions related to their appropriate use in routine practice depending on the virus distribution and heterogeneity within plants and on the appropriate sampling time and protocol.

\section{Materials and methods}

Tissue culture

New germplasm to be integrated in the collection were received at the ITC in Leuven, Belgium as suckers or in vitro plants. The set of samples received for a new plant was called a 'germplasm line'.

Explant materials received by the ITC were surface-disinfected before the start of culture. Of each individual sucker received, a tissue culture line was initiated from a small sterile plant apex (about $5 \mathrm{~mm}$ ). A bacteriological test was performed to detect endophytes in tissue culture lines multiplied for the first time at the ITC (Van den houwe \& Swennen, 2000). Each germplasm line was given a unique ITC identifier before commencement of full indexing and was then called an 'accession'.

Indexed healthy accessions conserved in the collection are maintained as in vitro cultures (Van den houwe et al., 1995), with a safety back-up preserved in liquid nitrogen (Panis et al., 2007).

\section{Full indexing of ITC accessions}

Full virus indexing was conducted on 243 accessions received as tissue culture plantlets between 2007 and 
2013, and consisted of specific molecular assays (described below) for BBTV, BBrMV, CMV, BanMMV and six BSV species, and an immunosorbent electron microscopy (ISEM) assay to check for any viruses. ISEM, as practiced in full indexing, combines the advantages of enhanced detection of additional BSV species, as the antiserum used is very cross reactive within the genus, while at the same time not preventing the detection of unrelated viruses.

Plant growth and indexing controls: Four in vitro rooted plantlets per accession were deflasked and grown in 25 $\mathrm{cm}$ diameter pots in post entry quarantine glasshouses in Brisbane, Australia. Infected controls for CMV, BBTV, Banana streak OL virus (BSOLV), the tentative species Banana streak CA virus (BSCAV), Banana streak MY virus (BSMYV), Banana streak IM virus (BSIMV) and Banana streak GF virus (BSGFV; the latter two a mixed infection) were grown in banana in a separate insect-proof glasshouse. Uninfected plants were grown in a third glasshouse under similar conditions. The BBrMV infected control (DAF Plant Virus Collection isolate Q509, from Philippines) was a mixed infection with BanMMV, and was stored at $-80^{\circ} \mathrm{C}$. As well as tissue positive controls, a mixture of plasmids containing appropriate sequences from BSOLV, BSCAV, BSMYV, BSIMV, BSGFV and the tentative species Banana streak Lacatan virus (BSV-Lac) was also included in the multiplex PCR assay (details below).

Subsampling and leaf extract preparation: At three and six months after deflasking, plants were observed for disease symptoms, and leaf samples were collected from the three youngest leaves of each of four plants per accession. These leaf samples were bulked together for testing. The midrib and lamina from each leaf sample were subsampled with biopsy punches; a total of $2 \mathrm{~g}$ for the partial purification and concentration procedure (viral miniprep) conducted ahead of the ISEM (see below), and a total of $0.1 \mathrm{~g}$ for the IC-(RT)-PCR assays.

Infected and uninfected controls were sampled (symptomatic tissue where possible and appropriate) following sampling of the indexing material. For the infected controls, two serial 1 in 10 dilutions in a healthy extract were prepared to confirm that assay sensitivity sufficiently allowed for bulking together of the four plants of each accession.

The IC-(RT)-PCR subsamples were ground using a TissueLyser (Qiagen, Hilden, Germany) in $1 \mathrm{ml}$ of CEB buffer (Sharman et al., 2000) and clarified by centrifuging at $10000 \mathrm{~g}$ for $5 \mathrm{~min}$.

\section{Molecular tests (IC-(RT)-PCRs):}

BBTV, CMV, BBrMV and BanMMV: The BBTV/CMV/ BBrMV multiplex IC-(RT)-PCR was conducted essentially as described by Sharman et al. (2000). For completeness, the details are given in Appendix S1, Supporting
Information. An additional specific PCR for BanMMV was conducted on the cDNA/BBTV DNA mixture generated for the multiplex PCR. Primers used are listed in Table 1. The PCR products were separated by electrophoresis in a $1.5 \%$ agarose gel in $0.5 \times \mathrm{TBE}$, and were stained with ethidium bromide.

Banana streak viruses: The BSV multiplex IC-PCR assay tested for six species of BSV: the three known activatable BSVs (BSIMV, BSGFV, BSOLV), another common and possibly integrated and activatable BSV (banana streak MY virus, BSMYV), and two other species within the BSV species complex (BSCAV and BSV-Lac) (A. Geering, personal communication) for which positive control material was available at the time and place of testing. ISEM (below) was used to index for other BSV species not amplified in this assay.

The immunocapture step for the BSV multiplex IC-PCR assay followed a similar format to the BBTV/CMV/BBrMV multiplex assay, except that reaction tubes were coated with polyclonal antibodies which cover a broad-spectrum of BSV species (see Appendix S1) and BSV specific primers were used in the PCR (Table 1). Additionally, a DNaseI treatment of the tubes was undertaken between the immunocapture and PCR steps of the assay to remove host DNA bound to the reaction tubes which may have contained endogenous BSV sequences and leave only encapsidated, episomal BSV nucleic acid for amplification (if present).

To check accessions for viruses not detected by the specific molecular assays (for instance sequence variants of known viruses, or novel viruses), partially purified and concentrated viral minipreps were prepared essentially as described by Lockhart (1986) and applied to electron microscopy grids coated with a mixture of polyclonal antibodies to enrich trapping of virus particles of known species which may not have been amplified by PCR (particularly BSV species), ahead of staining and viewing under a transmission electron microscope. Use of antibody coated grids does not preclude the binding and detection of virions which do not react with the coating antibodies. For completeness, the details are given in Appendix S2.

\section{Pre-indexing of germplasm lines}

This step, implemented from 2009, has the objective to quickly screen for germplasm lines requiring virus elimination before the full indexing step was undertaken, reducing the need for post-entry therapy and repeated virus indexing. It was optimised and carried out for 270 germplasm lines (434 samples) received as tissue culture plantlets and/or leaves from the ITC between 2009 and 2013. During the optimisation phase, samples 


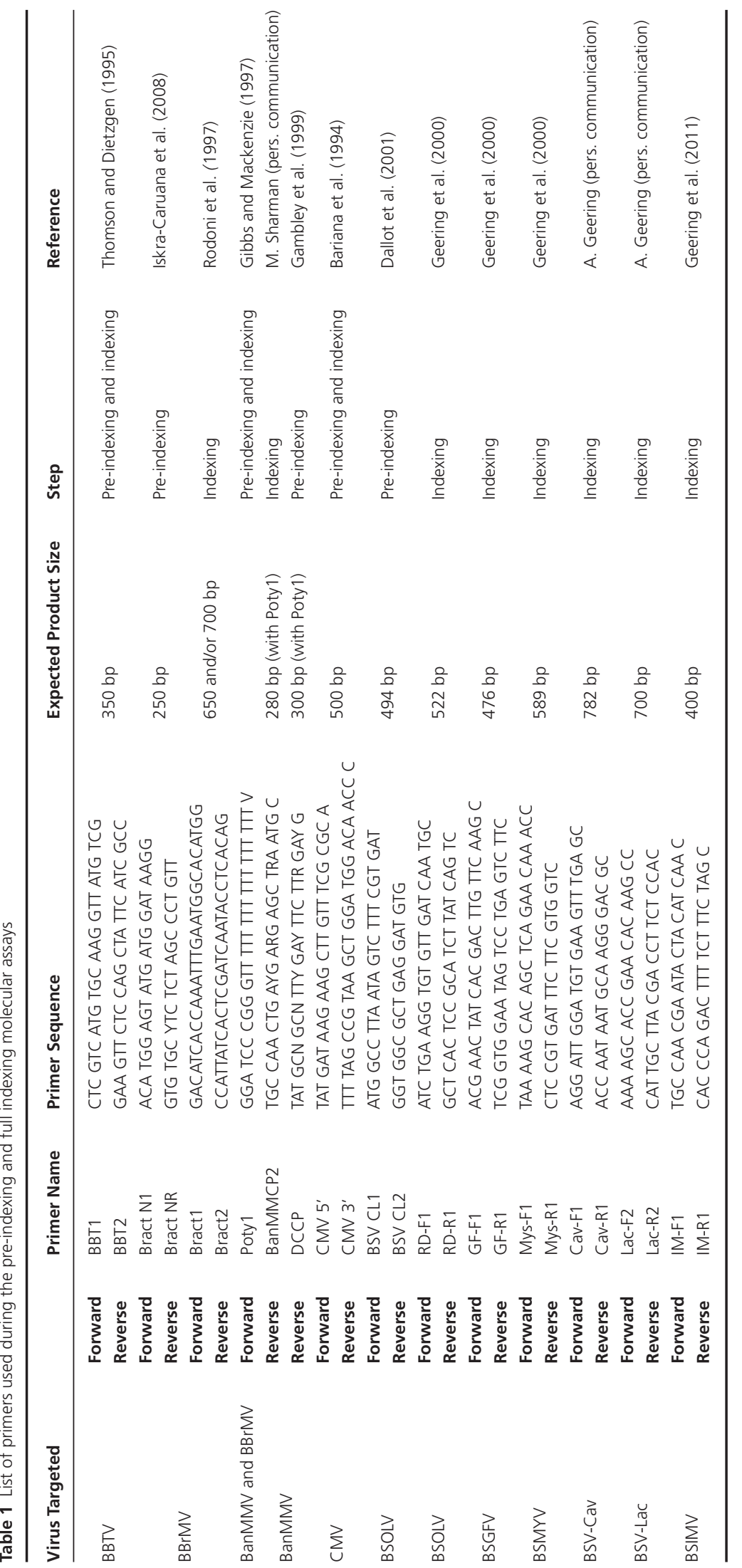


were tested as in vitro plantlets and in vivo plantlets after 1, 3 and 6 months of acclimatisation in the greenhouse, using specific molecular assays (described below) for the detection of BBTV, BBrMV, CMV, BanMMV and BSOLV.

\section{Plant growth and pre indexing controls}

Rooted in vitro plantlets were deflasked and grown in 25 $\mathrm{cm}$ diameter pots in Gembloux, Belgium, initially in a humid chamber ( $90 \%$ relative humidity) at a temperature of $23 \pm 2^{\circ} \mathrm{C}$ and a 16 -h photoperiod, and then after three weeks, in a heated insect-proof greenhouse with the same conditions of temperature and photoperiod. Infected controls for CMV, BBTV, BBrMV, BanMMV and BSOLV (origins unknown) were grown in banana in an insect-proof glasshouse. Uninfected plants were grown in a separate chamber of the glasshouse under similar conditions.

\section{Regular sampling and extraction}

For BBTV, CMV, BBrMV and BanMMV, $0.5 \mathrm{~g}$ of the youngest banana leaf (cigar leaf whenever possible) per plant was ground in $2 \mathrm{~mL}$ of extraction buffer ( $137 \mathrm{mM}$ $\mathrm{NaCl} ; 8 \mathrm{mM} \mathrm{Na} \mathrm{HPO}_{4} ; 1.5 \mathrm{mM} \mathrm{KH} \mathrm{PO}_{4} ; 2.7 \mathrm{mM} \mathrm{KCl} ; 80$ $\mathrm{mM} \mathrm{Na} 2 \mathrm{SO}_{3} ; 3 \mathrm{mM} \mathrm{NaN}_{3} ; 0.05 \%$ Tween 20, at $\mathrm{pH}$ 7.2; sterilised by filtration). For BSV detection, $0.5 \mathrm{~g}$ of the youngest banana leaf (cigar leaf if possible) per plant was ground in $2 \mathrm{~mL}$ of CEB buffer (Sharman et al., 2000).

\section{Molecular tests}

The molecular tests were carried out for BBTV, BBrMV, CMV, BanMMV and BSOLV using the protocols detailed in Appendix S3 and the primers listed in Table 1. For the five viruses, the PCR products were separated by electrophoresis in a $1.5 \%$ agarose gel in $0.5 \times \mathrm{TBE}$, and were stained with ethidium bromide.

\section{Complete procedure}

The final procedure of pre-indexing, indexing and sanitation of the banana germplasm accessions involved three partners who collaborated following the scheme detailed in Fig. 2. Each individual sample received at ITC Leuven was cultured in vitro and individually pre-indexed for five viruses through molecular techniques at Gembloux. A sucker which tested negative or the plantlet with the highest health status was then given an ITC accession number.

After in vitro multiplication, four clones of each accession were sent to Brisbane for full indexing, including symptoms observation, electron microscopy and molecular tests. Accessions in which no viruses were detected after full indexing were then made available for distribution by the ITC. ITC accessions in which virus(es) were detected entered the virus elimination process (described elsewhere).

\section{Results}

Detection of virus infections in pre- and full indexing results

The detailed full indexing results for each of 243 accessions are given in Appendix S4. Viruses were not detected in $68 \%$ of accessions (Fig. 3), and BBTV, CMV and BBrMV were not detected in any accession. BanMMV was detected in 46 accessions (from Honduras, India, Papua New Guinea, Tanzania, Uganda, Vietnam, or of unknown origin), and BSV in 44 accessions (from Cameroon, India, Indonesia, Papua New Guinea, Tanzania, Vietnam, or of unknown origin); 23 of these accessions were mixed infections of a BSV and BanMMV. Unidentified viruses (isometric, rod-shaped or Closteroviridae-like particles) were observed by TEM in 16 accessions (from India, Indonesia, Papua New Guinea, Tanzania, Thailand, Vietnam, or of unknown origin), in some cases as mixed infections with a BSV and/or BanMMV.

During the full indexing process, BSOLV was the most commonly detected BSV species, with 32 of 44 accessions $(72.7 \%)$ positive for this virus. Other detected BSV species were BSGFV (four accessions) and BSIMV (four accessions). In four accessions which tested negative by PCR for the six known BSV species, unidentified bacilliform (BSV-like) particles were observed by electron microscopy, highlighting the importance of the non-specific ISEM assay.

Analysis of the 434 samples (representing 270 germplasm lines) through the pre-indexing protocol revealed a high prevalence of virus infection (Fig. 4). The detailed pre-indexing results for each sample are given in Appendix S5. Viruses were not detected in 32.4\% of the tested samples. Samples infected with BSOLV and/or BanMMV represented $66.9 \%$ of the samples, amongst which one third were mixed infections. The presence of BanMMV and BSOLV infections was $47.8 \%$ and $43.9 \%$ respectively, while the presence of BBrMV and BBTV infections was $1.8 \%$ and $0.8 \%$ respectively. CMV was not detected in the tested samples. At the end of the pre-indexing process, 203 germplasm lines were assigned ITC accession numbers. The remaining 67 lines were assigned to virus elimination processes.

Amongst the 243 fully indexed accessions, 68 had also passed the preliminary pre-indexing step (that was introduced relatively recently to the full indexing programme), which allowed comparison of the results obtained in both steps (see Appendix S4). Twenty eight accessions indexed 


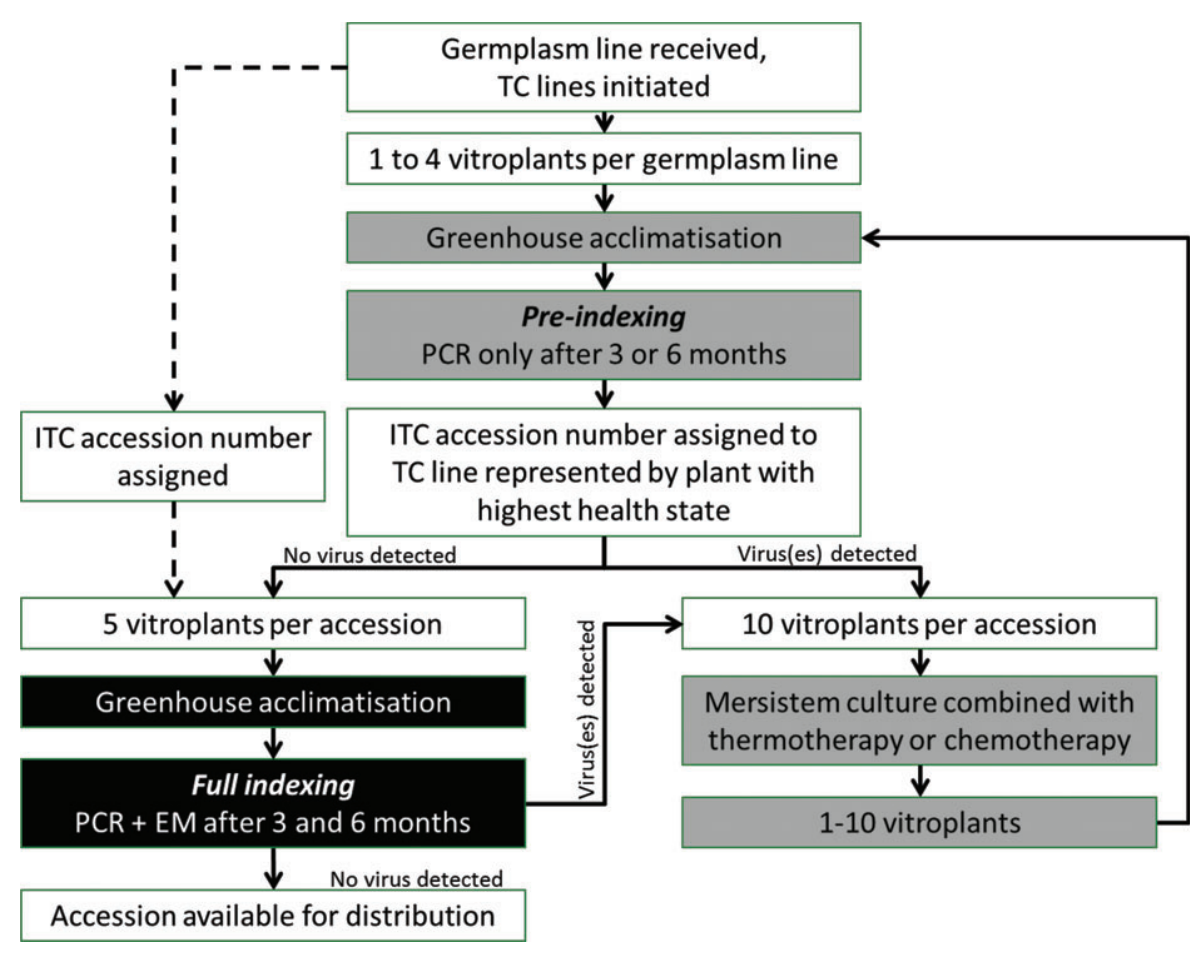

Figure 2 Indexing scheme for banana accessions of the ITC collection. Collection management (in white) was done by ITC, pre-indexing and sanitation processes (in grey) were carried out by Gembloux Agro-Bio Tech, and full indexing of the accessions (in black) was carried out by the Department of Agriculture and Fisheries (Queensland), Brisbane. The dotted line indicates the route for germplasm lines which did not undergo pre-screening.

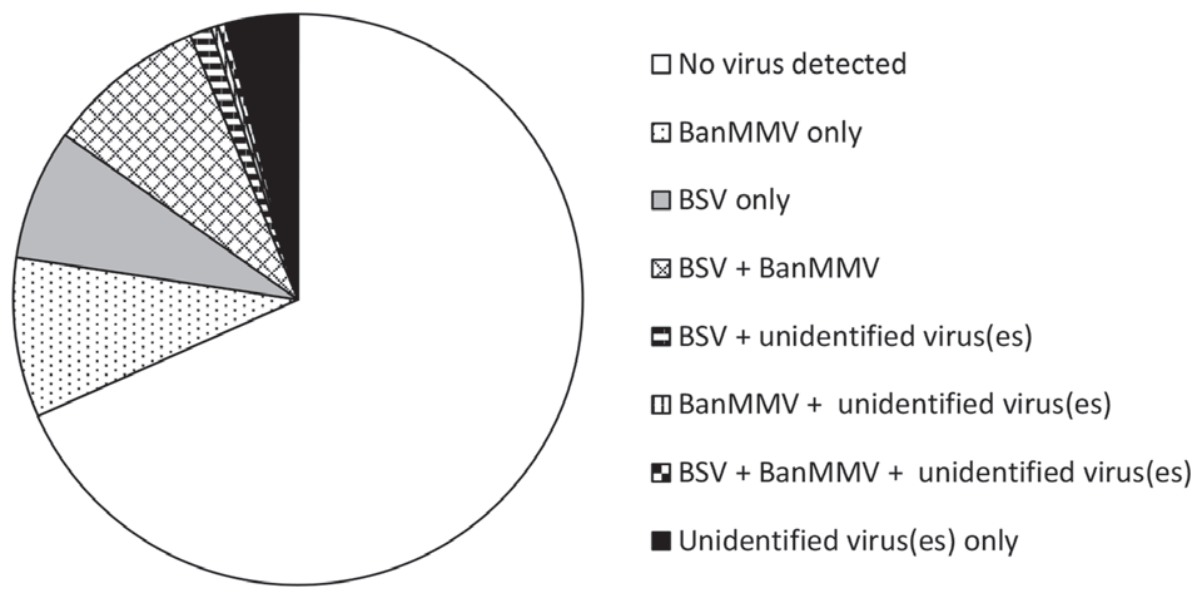

Figure 3 Relative proportions of viruses detected in 243 accessions during the full indexing step. Detections were: no virus detected (white), 166 accessions; BanMMV only (dots), 22; BSV only (grey), 18; BSV and BanMMV (cross-hatched), 22; BSV and unidentified virus(es) (thick stripes), 3; BanMMV and unidentified virus(es) (thin stripes), 1; BSV and BanMMV and unidentified virus(es) (checks), 0; and unidentified virus(es) only (black), 10. The viruses detected shown in the key are displayed in the same order in a clockwise direction on the pie chart.

negative and 12 indexed positive as well in pre-indexing and in full indexing. However, viruses (mostly BSV and BanMMV) were detected through full indexing of 23 accessions derived from pre-indexing samples which tested negative. These differences could be partly explained by the additional use of electron microscopy and the use of PCR assays for additional BSV species in full indexing, and by BSV activation (during tissue culture of the accession between the indexing steps or selection of individual plantlets in which BSV had been activated). For 11 of these 23 accessions, several samples were tested independently during the pre-indexing process and only 


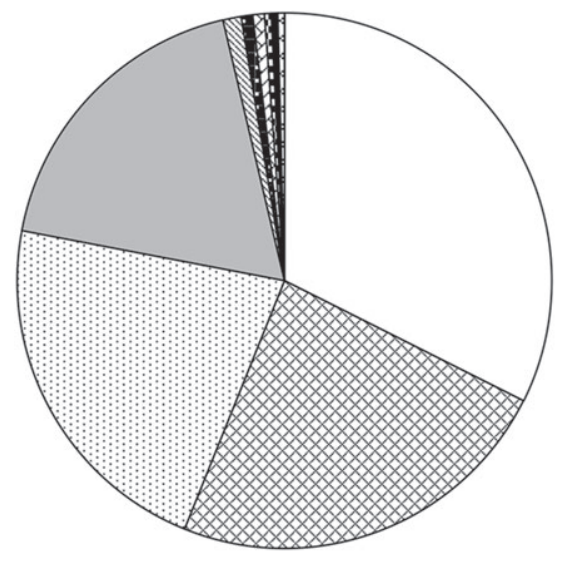

$\square$ No virus detected

BanMMV + BSOLV

๑BanMMV

$\square B S O L V$

BanMMV + BSOLV + BBrMV

- BanMMV + BBrMV

BSOLV + BBrMV

DBBTV

- BBrMV

BanMMV + BBTV

Figure 4 Relative proportions of viruses detected in 278 accessions during the pre-indexing step. Detections were: no virus detected (white), 90 accessions; BanMMV only (black dots), 61; BSOLV only (grey), 51; BSOLV and BanMMV (cross-hatched), 66; BanMMV, BSOLV and BBrMV (diagonal), 3; BanMMV and BBrMV (white dots), 2; BSOLV and BBrMV (bricks), 2; BBTV only (checks), 1; BBrMV only (black), 1; BanMMV and BBTV (tiles), 1. The viruses detected shown in the key are displayed in the same order in a clockwise direction on the pie chart.

some of them were negative. These negative samples were included in the collection as ITC accessions but later tested positive during full indexing. Five accessions which indexed positive during pre-indexing later indexed negative during full indexing, because individuals of highest health were included in the ITC collection and/or because of variable BSV activation.

\section{BSV infection and the B genome}

The detection of episomal BSV during pre-indexing and full indexing analyses was compared between banana genotypes (Fig. 5). Higher rates of BSV infection were detected in B-genome containing genotypes $(58 \%$ for pre-indexing and $40 \%$ for full indexing) compared with accessions lacking the $\mathrm{B}$ genome (12\% for pre-indexing and $3 \%$ for full indexing). No clear difference in the percentage of ITC accessions which tested positive for BSV was observed between genotypes containing the $\mathrm{B}$ genome $(\mathrm{BB}, \mathrm{AB}, \mathrm{BBB}, \mathrm{ABB}, \mathrm{AAB}, \mathrm{AAAB}, \mathrm{AABB}$, $\mathrm{ABBB})$.

Importance of greenhouse cultivation and sampling at several time points during growth

Indexing results for individual plants of germplasm lines infected with BanMMV or BSOLV were retrospectively analysed to evaluate the reliability of testing in vitro material and the effect of sampling time in greenhouse on virus detection. Detection of both BanMMV and BSOLV under pre-indexing conditions and protocols was
A

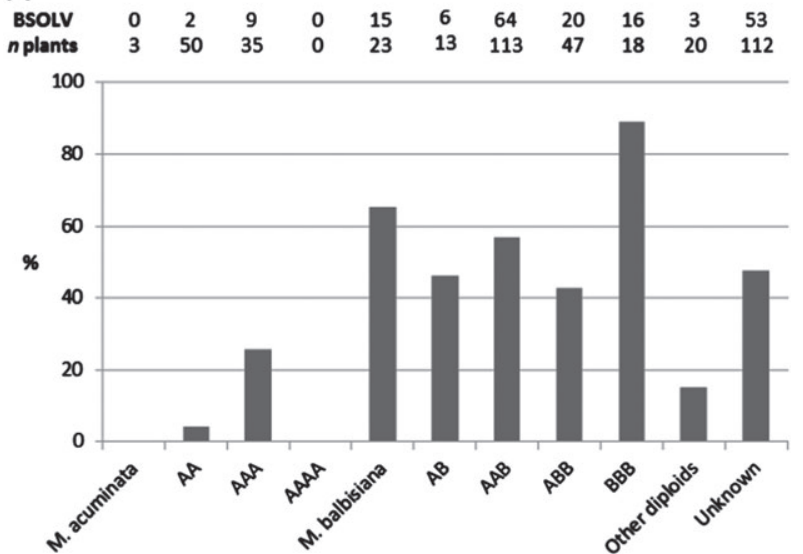

B

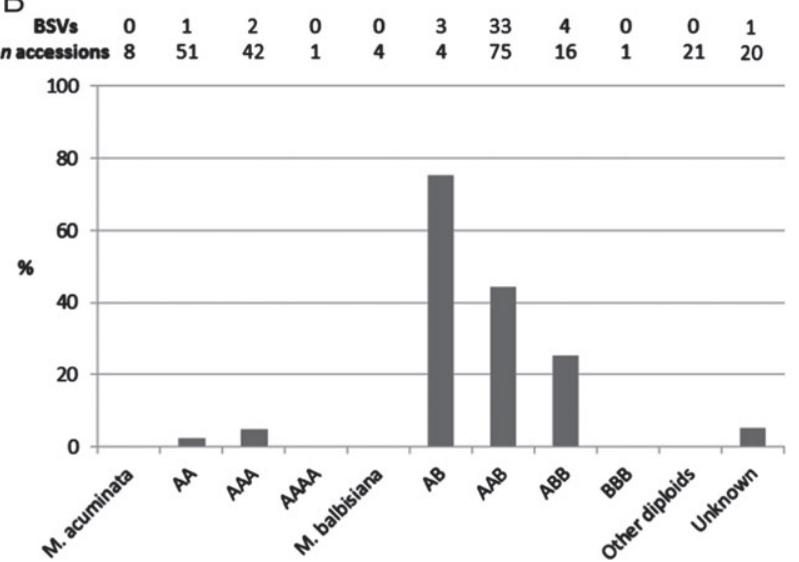

Figure 5 Percentage of BSV-positive accessions in different host genotypes. A) BSOLV detected through the pre-indexing process, B) BSV species detected through the full indexing process. Numbers of BSV detections and total plants tested per genotype are given above the respective percentage.

influenced by the sampling time. Of 15 plants known to be infected with BanMMV, only $20 \%$ of them tested positive in vitro, $27 \%$ after one month of greenhouse growth, 53\% after three months of greenhouse growth and $100 \%$ after six months of greenhouse growth. Of eight BSOLV infected plants, $37.5 \%$ tested positive in vitro, $75 \%$ after one month and $100 \%$ after three months of greenhouse growth.

Detection of BanMMV by PCR under full indexing conditions and protocols was reasonably reliable, with $80 \%$ of accessions testing positive at both three and six month sampling times (Fig. 6). However, only 55\% of accessions which tested positive for any BSV by PCR were detected at both sampling times. Detection of novel BSVs and unidentified viruses by ISEM was most variable between the two sampling times. These results strongly support the need for testing of the two sampling times for most comprehensive detection of virus infections. 


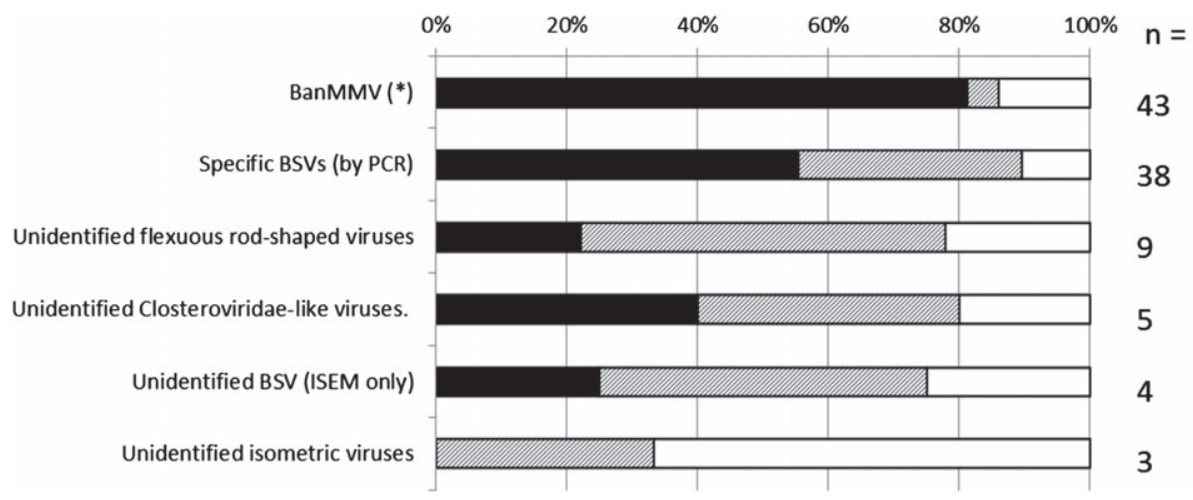

- Positive 3 and 6 months

口 Positive 3-months only

口Positive 6-months only

Figure 6 Consistency of virus detection between the 3 and 6 month sampling points during the full indexing process. Viruses were (1) BanMMV*, (2) Specific BSVs (PCR), (3) Unidentified BSVs (ISEM only), (4) Unidentified flexuous rod-shaped viruses, (5) Unidentified isometric viruses, and (6) Unidentified Closteroviridae-like viruses. *Three BanMMV-positive accessions tested only at 6 months were not included.

Table 2 Concordance of virus detection by pre-indexing protocol between several plants from the same accession ( $n=84$ accessions)

\begin{tabular}{llc}
\hline Status & Result & $n$ \\
\hline Concordance of result & No virus detection & 19 \\
& BSV & 16 \\
& BanMMV & 6 \\
& BSV and BanMMV & 9 \\
& Other combination & 3 \\
Discordance between results & BSV only & 11 \\
& BanMMV only & 12 \\
& BSV and BanMMV & 6 \\
& Other combination & 2 \\
\hline
\end{tabular}

Importance of testing several plants per accession

To investigate uniformity of virus infection in clonal plants, the pre-indexing results were analysed for 84 germplasm lines with ITC accession numbers (181 individually tested plants of which there were two to four plants per accession) grown and sampled at the same time under the same conditions (Table 2).

Identical results for each plant within a germplasm line were obtained for 53 lines (63\%). Among these, no virus was detected in 19 lines, while 16 were found to be infected by BSOLV (19\%), six by BanMMV alone (7\%), nine by a combination of both BSOLV and BanMMV $(11 \%)$, and three by other viruses $(2 \%)$. Discordant results between plants within a germplasm line were obtained for 31 lines $(37 \%)$.

When analysing the results by viral species, BanMMV was detected in 37 germplasm lines (61 plants). Among them, discordant results were observed for 17 lines $(46 \%)$. BSOLV was detected in 41 germplasm lines (77 plants) and discordant results were observed for 17 lines $(41 \%)$.

\section{Discussion}

\section{Virus distribution}

This large scale study allowed interesting observations about the distribution of major banana viruses worldwide. The detection of viruses in the field-sampled plants can be compared to the status of the viruses in these countries. The most frequently detected viruses, BanMMV and BSV, have a worldwide distribution and were detected in samples originating from Asia, Africa, Oceania and Central America (India, Malaysia, Vietnam, Cameroon, Uganda, Honduras and Papua New Guinea). Importantly, we have not identified new outbreaks of viruses; the plants that we detected as being infected originated from countries where both viruses have already been recorded.

In our study, very few samples from geographical areas where BBTV is present were infected by the virus. This fact can be explained by the objective of the sampling, which was to obtain high health status material for inclusion in germplasm collections. Indeed, the symptoms of the disease are readily identified in most cultivars and asymptomatic infected plants are rare. It was shown that ninety percent of the farmers from the Great Lake Region were able to recognise bunchy top symptoms (Niyongere et al., 2012). Moreover, the process of initiating the plants in vitro can eradicate the virus (Lassois et al., 2013), further reducing the chance of introducing BBTV into collections. CMV was not detected in our study, neither in pre-indexing nor indexing steps. Here again, it can most likely be explained by the fact that the symptoms of the disease are generally easy to recognise. BBrMV has a limited distribution worldwide and infected plants can display obvious symptoms, which could likewise explain why it was rarely detected in our study. BanMMV is widely distributed worldwide but expression of symptoms of the disease is infrequent. This can explain the 
high prevalence that we observed for this virus. However, BanMMV presence in the germplasm collection must be avoided to prevent emergence of more virulent strains and to minimise the risk of unpredictable variations in symptoms, infectivity, accumulation and/or vector transmissibility due to co-infection with other banana viruses.

We observed the presence of BSOLV in $44 \%$ of the pre-indexed plants. The BSV infection was highly correlated with $\mathrm{B}$ genome presence in the accession (10 times more prevalent than accessions without the B genome). This difference is probably due to the activation of viral DNA integrated within the $\mathrm{B}$ genome of banana. The testing scheme itself can induce the expression of integrated BSV as a micropropagation procedure known to activate BSV expression (Dallot et al., 2001) is applied before pre-indexing and/or indexing. This is a significant issue in the movement of accessions with the B genome and the consequent risk associated with the activation of the integrated BSV (Bioversity, 2015). The absence of difference in BSV detection between genotypes containing B genome is surprising, since some studies have raised the hypothesis that hybrids containing two copies of the B genome could not be infected by BSV (Chabannes et al., 2013).

\section{Lessons learned for indexing}

This study reports the results of a two-step process, where a pre-indexing step was implemented in order to save time overall. This step potentially allowed the selection of healthy plants within infected germplasm lines and early implementation of a sanitation process in $68 \%$ of lines. The accessions that went through both pre-indexing and full indexing recorded similar virus detection results, showing the reliability of the methods used. The only observed dissimilarities so far could be attributed to the increased efficiency of indexing with the use of TEM in indexing, the inclusion of additional PCR assays for BSV species, or the potential for BSV activation in accessions containing B genomes. This suggests a strong complementarity between steps and shows the utility of this sequential testing.

Our results also showed that the analysis of multiple plants for a single accession should be recommended in order to lower the false negative rate. The variability of detection between individual plants can be due to a heterogeneous distribution of the virus in the plant, differing virus titers between plants, the activation of BSV or to fortuitous elimination of the virus during in vitro propagation. The independent testing of several plants per accession improves the reliability of indexing but also significantly raises the cost of diagnostic analysis.
Combining samples from several plants of a single accession to have a pooled sample reduces these costs, but it is critical that suitable dilutions of positive controls are included to demonstrate that test sensitivity remains adequate. The growing of several plants per accession and indexing as a pooled sample is practiced routinely for the full indexing step, as is the use of 10- and 100-fold dilutions of the tissue positive controls during PCR assays.

Testing in vitro plants for the presence of viruses was an option worth investigating to determine whether the virus status of an in vitro maintained accession could be reliably assessed without the need to grow plants in a greenhouse. However, the false negative rate of in vitro samples during pre-indexing was particularly high and therefore the testing of in vitro samples should not be used as the sole method of indexing. Greenhouse cultivation of the in vitro plants is then mandatory and an acclimatisation period of 6 months seems to be needed for efficient detection of BSV and BanMMV. Other ways of reducing the analysis period are now being evaluated by the partners within Bioversity. These include adapting the guidelines to allow indexing of leaf or pseudostem material sampled from the mother plant at the time of collection or at the point of in vitro establishment.

The ability to perform a general viral and viroid indexing on a plant sample, including the detection of distant variants of known viruses or unknown or novel agents is an extremely attractive possibility in this particular situation. The full indexing of banana accessions is made through a combination of IC-(RT)-PCR and electron microscopy. It is well known that the molecular techniques based on PCR are more sensitive than electron microscopy to detect the presence of viruses. However, the application of molecular or immunological techniques is largely restricted to known and recently well characterised viral agents for which serological reagents and/or sequence information are available. For unknown agents or those still too poorly characterised, the diagnostician still faces very complex challenges that are partially met by the use of polyvalent serological or molecular assays or by the use of electron microscopy or symptomatology. This is the reason why molecular tests are complemented by TEM and observation for virus symptoms. Such a combination can be currently considered as mandatory as unidentified viral particles were observed in 22 accessions for which no virus was detected by molecular techniques. These results need further investigation but four hypotheses could explain the observed results and are under study: (i) the virus distribution is heterogeneous in the plant and viral particle were only detectable in the sampling for TEM, (ii) the observed viruses are other less common viruses of banana (like Sugarcane 
mosaic virus for example), (iii) the observed viruses are divergent strains of the tested species with mutation in the primer annealing sites and (iv) the observed viruses are not yet known or have never been reported on banana. An approach combining polyvalent PCR primers targeting virus families and high-throughput sequencing should narrow the hypotheses and allow better understanding of negative results of the PCR.

Nevertheless, the false negative rate of TEM was above $30 \%$ for BanMMV and BSV, precluding its use as the sole detection method for these viruses. As a consequence a full virological indexing, i.e. the identification of all viruses present in a given sample, is still a difficult objective. Recent developments in high-throughput sequencing (or Next Generation Sequencing - NGS) technologies and in bioinformatic analyses of the vast amount of sequence data thus generated have changed this situation drastically. Indeed, it is now conceptually feasible to detect any viral agent by high-throughput sequencing of the nucleic acids from a host and the identification of viral sequences of known or unknown agents in the generated sequences (Massart et al., 2014). The indexing of Musa accessions could therefore benefit in the future from the advances in NGS application to diagnostics, though distinguishing episomal from endogenous BSV sequences will represent an important consideration. The application of these innovative indexing protocols can be considered as a key development in order to achieve a complete virus indexing of the accessions, advantageously replacing the actual combination of molecular tests and TEM. However, caution is necessary, as the inconsistent application of high sensitivity assays to samples from some countries only, and the possible recognition of novel viruses, could have serious implications for quarantine and the international movement of germplasm.

In conclusion, the high percentage of infected plants originating from the field (68\% in pre-indexing) underlines the importance of evaluating virus presence in accessions before their distribution. A comprehensive scheme including pre-indexing and full indexing, and combining various diagnostic protocols currently gives the highest chance for detection of viruses in the accessions and the safe release of high health status accessions. Even if the distribution of infected accessions is a recurrent debate among the scientific community, more particularly for BSV (Bioversity, 2015), complete virus indexing as currently carried out will remain mandatory to characterise the viral status of any accession. We can also anticipate that the rise of NGS technologies in the plant virus diagnostic field will bring new opportunities to improve significantly the efficiency and the reliability of the indexing of Musa accessions.

\section{Acknowledgements}

The authors would like to thank Angelo Locicero for his technical assistance, David Spence for maintenance of plants in post-entry quarantine and Andrew Geering for sharing technical protocols for BSV indexing. We would like to acknowledge the support of the Belgian Development Cooperation (DGD) and Horticulture Innovation Australia Limited. We would also like to thank all donors who supported this work through their contributions to the CGIAR Fund (http://www.cgiar.org/who-weare/cgiar-fund/fund-donors-2/), and in particular to the CGIAR Research Program for Managing and Sustaining Crop Collections (Genebanks-CRP).

\section{References}

Bariana H.S., Shannon A.L., Chu P.W.G., Waterhouse P.M. (1994) Detection of five seedborne legume viruses in one sensitive multiplex polymerase chain reaction test. Phytopathology, 84, 1201-1205.

Bioversity (2015) Final Position Paper on the strategy to distribute Banana Streak Virus (BSV) infected germplasm [Online]. Bioversity International. URL https://sites.google.com/a/cgxc hange.org/musanet/news/finalpositionpaperonthestrategy todistributebananastreakvirusbsvinfectedgermplasm

Blomme G., Ploetz R., Jones D., De Langhe E., Price N., Gold C., Geering A., Viljoen A., Karamura D., Pillay M., Tinzaara W., Teycheney P.-Y., Lepoint P., Karamura E., Buddenhagen I. (2013) A historical overview of the appearance and spread of Musa pests and pathogens on the African continent: highlighting the importance of clean Musa planting materials and quarantine measures. Annals of Applied Biology, 162, 4-26.

Chabannes M., Baurens F.-C., Duroy P.-O., Bocs S., Vernerey M.-S., Rodier-Goud M., Barbe V., Gayral P., Iskra-Caruana M.-L. (2013) Three infectious viral species lying in wait in the banana genome. Journal of Virology, 87, 8624-8637.

Cook D.C., Liu S., Edwards J., Villalta O.N., Aurambout J.P., Kriticos D.J., Drenth A., De Barro P.J. (2012) Predicting the benefits of banana bunchy top virus exclusion from commercial plantations in Australia. PLOS ONE, 7, e42391. DOI:10.1371/journal.pone.0042391.

Dallot S., Acuna P., Rivera C., Ramirez P., Cote F., Lockhart B.E., Caruana M.L. (2001) Evidence that the proliferation stage of micropropagation procedure is determinant in the expression of banana streak virus integrated into the genome of the FHIA 21 hybrid (Musa AAAB). Archives of Virology, 146, 2179-2190.

Diekmann M., Putter C. (1996) FAO/IPGRI Technical Guidelines for the Safe Movement of Germplasm. No. 15. Musa. 2nd edn. Rome, Italy: FAO/IGRI.

Duroy P.O., Perrier X., Laboureau N., Jacquemoud-Collet J.P., Iskra-Caruana M.L. (2016) How endogenous plant 
pararetroviruses shed light on Musa evolution. Annals of Botany, 117, 625-641.

FAO (2014) FAOSTATS 2012. Rome, Italy: FAO.

Frison P., Putter C. (1989) FAO/IBPGR Technical Guidelines for the Safe Movement of Musa Germplasm. Rome, Italy: FAO, IBPGR.

Garming H., Roux N., Van den houwe I. (2010) The Impact of the Musa International Transit Centre-Review of Its Services and Cost Effectiveness and Recommendations for Rationalization of its Operations. Montpellier: Bioversity International.

Geering A. D. W. (2009) Viral pathogens of banana: outstanding questions and options for control. In Proceedings of International ISHS-ProMusa Symposium on Recent Advances in Banana Crop Protection for Sustainable Production and Improved Livelihoods, White River, South Africa, 10-14 September 2007. Acta Horticulturae 828, pp. 39-50. Eds D.R. Jones and I. Van den Bergh. Leuven, Belgium: ISHS.

Geering A.D., McMichael L.A., Dietzgen R.G., Thomas J.E. (2000) Genetic diversity among banana streak virus isolates from Australia. Phytopathology, 90, 921-927.

Geering A.D., Parry J.N., Thomas J.E. (2011) Complete genome sequence of a novel badnavirus, banana streak IM virus. Archive of Virology, 156, 733-737.

Gibbs A., Mackenzie A. (1997) A primer pair for amplifying part of the genome of all potyvirids by RT-PCR. Journal of Virological Methods, 63, 9-16.

Iskra-Caruana M.-l., Galzi S., Laboureau N. (2008) A reliable IC One-step RT-PCR method for the detection of BBrMV to ensure safe exchange of Musa germplasm. Journal of Virological Methods, 153, 223-231.

Iskra-Caruana M.L., Chabannes M., Duroy P.O., Muller E. (2014) A possible scenario for the evolution of Banana streak virus in banana. Virus Research, 186, 155-162.

Kumar P.L., Selvarajan R., Iskra-Caruana M.L., Chabannes M., Hanna R. (2015) Biology, etiology, and control of virus diseases of banana and plantain. Advances in Virus Research, 91, 229-269.

Lassois L., Lepoivre P., Swennen R., van den Houwe I., Panis B. (2013) Thermotherapy, chemotherapy, and meristem culture in banana. In Thermotherapy, Chemotherapy, and Meristem Culture in Banana, pp. 419-433. Eds M. Lambardi, E.A. Ozudogru and S.M. Jain, Shri Mohan. Totowa, NJ, USA: Humana Press.

Lockhart B.E.L. (1986) Purification and serology of a bacilliform virus associated with banana streak disease. Phytopathology, 76, 995-999.

Massart S., Brostaux Y., Barbarossa L., César V., Cieslinska M., Dutrecq O., Fonseca F., Guillem R., Laviña A., Olmos A., Steyer S., Wetzel T., Kummert J., Jijakli M.H. (2008) Inter-laboratory evaluation of a duplex RT-PCR method using crude extracts for the simultaneous detection of Prune dwarf virus and Prunus necrotic ringspot virus. European Journal of Plant Pathology, 122, 539-547.

Massart S., Brostaux Y., Barbarossa L., Batlle A., Cesar V., Dutrecq O., Fonseca F., Guillem R., Komorowska B., Olmos
A., Steyer S., Wetzel T., Kummert J., Jijakli M.H. (2009) Interlaboratory evaluation of two reverse-transcriptase polymeric chain reaction-based methods for detection of four fruit tree viruses. Annals of Applied Biology, 154, 133-141.

Massart S., Olmos A., Jijakli H., Candresse T. (2014) Current impact and future directions of high throughput sequencing in plant virus diagnostics. Virus Research, 188, 90-96.

Niyongere C., Losenge T., Ateka E.M., Nkezabahizi D., Blomme G., Lepoint P. (2012) Occurrence and distribution of banana bunchy top disease in the Great Lakes Region of Africa. Tree and Forestry Science and Biotechnology, 6, $102-107$.

Ortiz R., Swennen R. (2014) From crossbreeding to biotechnology-facilitated improvement of banana and plantain. Biotechnology Advances, 32, 158-169.

Panis B., Van den houwe I., Piette B., Swennen R. (2007) Cryopreservation of the banana germplasm collection at the ITC (International Transit centre - Bioversity International). Advances in Horticultural Science, 21, 235-238.

Rodoni B.C., Ahlawat Y.S., Varma A., Dale J.L., Harding R.M. (1997) Identification and characterization of Banana bract mosaic virus in India. Plant Disease, 81, 669-672.

Sharman M., Thomas J.E., Dietzgen R.G. (2000) Development of a multiplex immunocapture PCR with colourimetric detection for viruses of banana. Journal of Virological Methods, 89, 75-88.

Thomas J.E., Sharman M., Lassois L., Massart S., De Clerck C., Caruana M.L., Chabannes M., Teycheney P.Y., Kumar P.L., van den Houwe I., Roux N. (2015) Musanet Technical Guidelines for the Safe Movement of Musa Germplasm. 3rd edn. Rome, Italy: Bioversity International.

Thomson D., Dietzgen R.G. (1995) Detection of DNA and RNA plant viruses by PCR and RT-PCR using a rapid virus release protocol without tissue homogenization. Journal of Virological Methods, 54, 85-95.

Van den houwe I., Swennen R. (2000) Characterization and control of bacterial contaminants in in vitro cultures of banana (Musa spp.). Acta Horticulturae, 530, 69-79.

Van den houwe I., Smet K., Montcel H.T., Swennen R. (1995) Variability in storage potential of banana shoot cultures under medium term storage conditions. Plant Cell, Tissue and Organ Culture, 42, 269-274

\section{Supporting Information}

Additional Supporting Information may be found in the online version of this article:

Appendix S1. Molecular detection tests of banana viruses used in full-indexing: detailed protocol.

Appendix S2. Immunosorbent electronmicroscopy of viral minipreps: detailed protocol.

Appendix S3. Molecular detection tests of banana viruses used in pre-indexing: detailed protocol. 
Appendix S4. Detailed results of virus detection obtained in full indexing. ITC code: code of the accession after integration in the ITC. 1: virus detected; 0: virus not detected.

Appendix S5. Detailed results of virus detections obtained in pre-indexing. In some cases, accessions already lodged with the ITC were pre-indexed and/or fully indexed, in which case the ITC accession number corresponded to the germplasm line identifier and all the tested plants kept the accession number (marked with a * in the table). Germplasm line: code for the line prior to its integration in the ITC; Sample: individual plant of a germplasm line; ITC accession number: accession number assigned to an individual plant of a germplasm line after integration in the ITC. 1 : virus detected; 0 : virus not detected. 